\title{
Effects of translational symmetry breaking induced by the boundaries in a driven diffusive system
}

\author{
Jørgen Vitting Andersen \\ Department of Structural Properties of Materials, The Technical University of Denmark, \\ Building 307, DK-2800 Lyngby, Denmark \\ Kwan-tai Leung \\ Department of Physics, Virginia Polytechnic Institute and State University, Blacksburg, Virginia 24061
}

(Received 19 December 1990)

\begin{abstract}
We study the effects of the boundary conditions in a driven diffusive lattice-gas model which is known to display kinetic phase transitions. We find, in the case of attractive interaction, that a boundary-condition-induced symmetry breaking of the translational invariance, along the direction of the external field, destroys the second-order kinetic phase transition. This feature is absent in equilibrium systems. In the repulsive case, the phase diagram and critical properties are probably unaltered.
\end{abstract}

Phase transitions and critical phenomena in systems in nonequilibrium steady state have attracted much attention in recent years. ${ }^{1-13}$ The primary interest is to investigate if and how the corresponding equilibrium properties are affected. In particular, one of the fundamental issues is to determine the quantities that are necessary and, if possible, sufficient to extend the concept of universality to systems in nonequilibrium steady states.

The major distinctive feature of a system in steady state is the existence of transport phenomena; these could be maintained by, for example, a temperature gradient, a chemical-potential gradient, or an external (electric) field in the respective cases of thermal, chemical, or electrical transport. Recently a nonequilibrium model proposed by Katz, Lebowitz, and Spohn has received extensive interest. $^{1-10,13}$ It is a stochastic lattice-gas model in which particles are biased to hop along an external electric field $E$ (hereafter called the KLS model). Not surprisingly, the critical behavior depends on the additional symmetry properties introduced by $E$, besides the usual criteria such as the symmetries of the order parameter and the spatial dimensionality. Thus, for instance, the breaking of the symmetry $J \rightarrow-J, \sigma_{i} \rightarrow(-1)^{i} \sigma_{i}$ (i.e., flipping every other spin) in the Ising Hamiltonian

$$
\mathscr{H}=-\frac{J}{4} \sum_{\langle i, j\rangle} \sigma_{i} \sigma_{j}
$$

by $E$ results, in the two cases of attractive ${ }^{6}(J>0$, ferromagnetic) and repulsive ${ }^{10}(J<0$, antiferromagnetic) interactions, in completely different phase diagrams and critical exponents. The sum is over nearest neighbors, and $\sigma= \pm 1$.

Along this line of thought, we investigate here by Monte Carlo simulation the effects of symmetry breaking by the boundaries in nonequilibrium steady state. This is stimulated by very recent studies of a model (hereafter called the boundary-driven model) in which the particles are driven only through the boundaries. ${ }^{11}$ In such a model, a nonequilibrium steady state is maintained provided the system size is finite. The resulting bulk properties are very different from their equilibrium counterparts. In stark contrast to equilibrium systems, where the bulk behavior is independent of the boundary conditions in the thermodynamic limit, we will see that the boundaries (normal to the flux direction) play a crucial role in determining the global, nonequilibrium steady-state properties.

It is not difficult to understand the importance of the boundaries in steady-state systems. As a trivial example, if we replace the periodic boundary conditions (PBC) in the KLS model with attractive interactions by those of a closed box, the system will simply settle into equilibrium with $E$ playing the equivalent role of gravity, and the flux is zero. At a more subtle level, the replacement of PBC by shifted (or screw) periodic boundary conditions in previous studies resulted in morphological transitions at low temperature not seen in equilibrium systems. ${ }^{9}$ Therefore, upon changing the boundary conditions, it is reasonable to expect modifications in the steady-state bulk properties that survive in the thermodynamic limit. The physical origin of this effect is conjectured to arise from long-range effects ${ }^{7}$ propagated into the bulk from the boundaries via the flux.

\section{MODEL}

Our kinetic lattice-gas model on a two-dimensional $L \times L$ lattice is defined by three elements: (i) The interaction among the particles through the usual lattice-gas Hamiltonian:

$$
\mathscr{H}=-J \sum_{\langle i, j\rangle} n_{i} n_{j}
$$

Here $J$ is the nearest-neighbor-coupling constant, the occupation variable $n_{i}=0,1$. (ii) The transition probability for going from one configuration to another, in which the electric field is dynamically imposed:

$$
P=\min \left\{1, \exp \left[-(\delta \mathcal{H}+\epsilon E) / k_{B} T\right]\right\},
$$

where $\delta \mathscr{H}$ is the energy difference between the two configurations, $E$ is the strength of the field which will be 
taken to be positive and uniform in the $x$ direction, while $\epsilon=-1,0,1$, depending on whether the particle jumps along, transverse to, or against the field direction. The dynamics we use is particle-hole exchange only between nearest neighbors. The overall density $\rho \equiv\left(1 / L^{2}\right) \sum_{i} n_{i}$ is fixed at $\frac{1}{2}$. (iii) The boundary conditions: In the direction transverse to $E, \mathrm{PBC}$ are used. The crucial physical feature for our driven system comes in through the boundary conditions at the two open boundaries at $x=1$ and $x=L$, transverse to the field. In order to conserve the total number of particles, a particle leaving the system through one edge always enters through the other. This is done without taking into account the $E$ field in (2). This treatment gives rise to a breaking in the translational invariance in the direction of the $E$ field. Our model is an extension of the boundary-driven model in Ref. 11, now with an $E$ field in the bulk. In the boundary-driven model, a current was maintained by asymmetric chemical potentials at two edges, corresponding to a local $E$ field acting between column 1,2 and between column $(L-1), L$. The reason for introducing a bulk $E$ field is to maintain a current in the thermodynamic limit. Without the bulk field, the current vanishes as $L^{-1}$, where $L$ is the length along the direction of the current. ${ }^{11}$ We will see that the steady-state properties are qualitatively different in the two cases.

Alternatively, our model can also be regarded as a variant of the KLS model. The only difference is the aforementioned boundary conditions along the $E$ field, instead of PBC used in the KLS model. The resultant weakening of flux across the boundaries as a result of the absence of $E$ is important, as we will see that it gives rise to dramatic changes in the kinetic phase transitions as compared to the KLS model.

\section{RESULTS}

To examine finite-size effects the Monte Carlo runs were done on $L \times L=8 \times 8,16 \times 16,32 \times 32,64 \times 64$, and $128 \times 128$ lattices. The simulations were done with up to $10^{6}$ Monte Carlo steps per site. The need for such long runs is due to strong fluctuations in the order parameter and energy, unseen in equivalent equilibrium systems. In order to determine whether a phase transition takes place in the driven system, we have calculated the specific heat $C_{L} \equiv \partial\left\langle u_{L}\right\rangle / \partial T, u_{L}$ being the energy per site of a system of size $L$, and the order parameter for the two $(J>0, J<0)$ cases. In the ferromagnetic case we define the order parameter to be the square root of the structure factor $\sum_{x, y} e^{i 2 \pi x / L}\left\langle\left(2 n_{x, y}-1\right)\left(2 n_{0,0}-1\right)\right\rangle$, after properly normalizing to unity at complete phase separation. For the antiferromagnetic case, the order parameter (staggered magnetization) is defined by $\langle|p|\rangle$, where $p \equiv\left(1 / L^{2}\right) \Sigma_{x, y}$ $\times(-1)^{x+y}\left(2 n_{x, y}-1\right)$. In equilibrium systems where one has a fluctuation-dissipation theorem, the specific heat $C_{L}$ can be calculated from the energy fluctuations $\left(\Delta u_{L}\right)^{2}$ $=\left\langle u_{L}^{2}\right\rangle-\left\langle u_{L}\right\rangle^{2}, C_{L}=L^{2}\left(\Delta u_{L}\right)^{2} / k_{B} T^{2}$, and the susceptibility corresponding to the order parameter is given by $\chi \equiv \partial p /\left.\partial H\right|_{H=0}=L^{2}(\Delta p)^{2} / k_{B} T$, where $H$ is an appropriate magnetic field that couples to the order parameter, and $(\Delta p)^{2}=\left\langle p^{2}\right\rangle-\langle|p|\rangle^{2}$ is the fluctuations in the order parameter. Even though we do not know if there is a fluctuation-dissipation theorem for the driven system, we find that in the case of repulsive interactions $(J<0) \Delta u_{L}$ and $\Delta p$ are useful indicators of where the phase transition takes place.

\section{Ferromagnetic $(J>0)$ case}

We find in the case of attractive interactions, and at low temperature $T$, a phase separation between a solid (density $\rho \approx 1)$ and a gas $(\rho \approx 0)$ phase with an interface perpendicular to the $E$ field. This behavior is in analogy to the boundary-driven model, but the current in our model is sustained in the limit $L \rightarrow \infty$. In the KLS model, the phase separation takes place with the interface parallel to the $E$ field. Because of PBC there is no broken translational invariance in the direction of the field. The KLS model undergoes a second-order phase transition and the critical temperature $T_{c}$ increases and eventually saturates with the field strength $E$.

The first difference from the KLS model is that the energy fluctuations $\left(\Delta u_{L}\right)^{2}$ have a distinct maximum as a function of temperature, whereas in the KLS model $\left(\Delta u_{L}\right)^{2}$ is a monotonic decreasing function of temperature, signaling a breakdown of the fluctuation-dissipation theorem. Comparing $\left(\Delta u_{L}\right)^{2}$ with $\partial\left\langle u_{L}\right\rangle / \partial T$ we find a difference in the two curves, which is greater than the error bars can account for, around the temperature at which the maximum occurs. This becomes more pronounced with growing $L$. Thus we cannot claim that the fluctuation-dissipation theorem is valid in our model. We have no explanation for the different behavior of $\left(\Delta u_{L}\right)^{2}$ in comparison to the KLS model.

Figure 1 shows the specific heat $C_{L}=\partial\left\langle u_{L}\right\rangle / \partial T$, obtained by numerical differentiation, as a function of temperature for different lattice sizes $L$ for $E=0.5 J$. The data represents simulations of $10^{6}$ Monte Carlo steps per site. The behavior of the maximum of $C_{L}$ is in stark contrast to the exact results for the Ising equilibrium model where the scaling relation $C_{L} \approx A+B \log L$ holds at

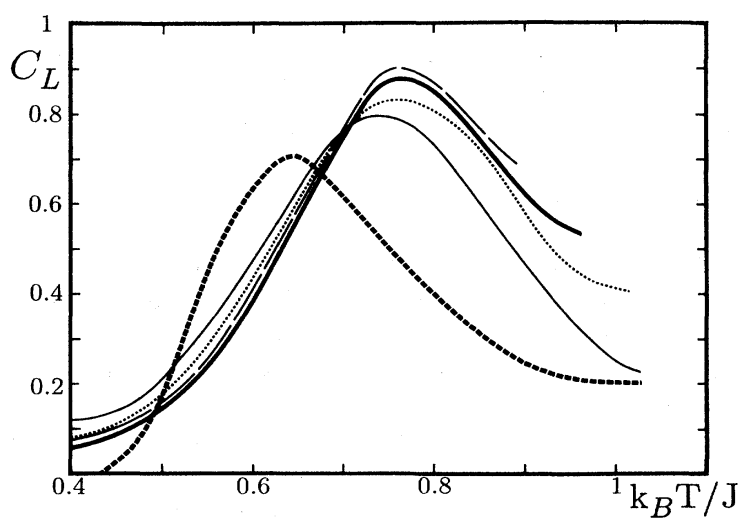

FIG. 1. Specific heat $C_{L} \equiv \partial\left\langle u_{L}\right\rangle / \partial T$, obtained by numerical differentation, vs temperature $k_{B} T / J$, for $E=0.5 J$. Five different system sizes $L \times L$ are shown: thick dots correspond to $8 \times 8$; thin line $16 \times 16$; thin dots $32 \times 32$; thick line $64 \times 64$; and dashed line $128 \times 128$. 


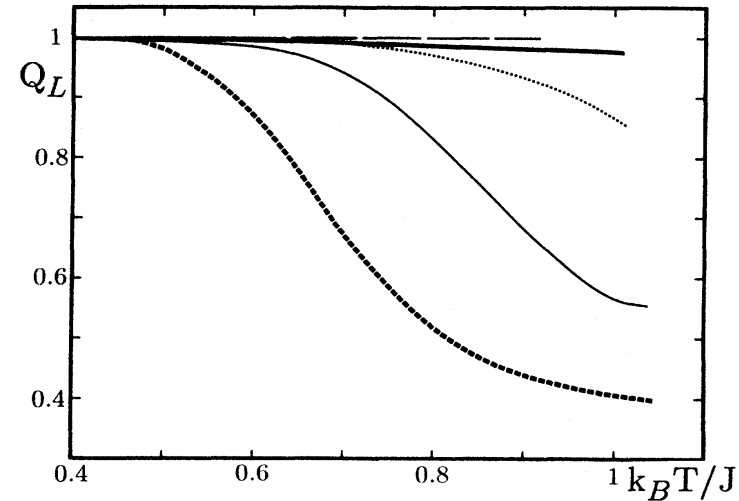

FIG. 2. Cumulant $Q_{L}=\left\langle p_{L}^{2}\right\rangle^{2} /\left\langle p_{L}^{4}\right\rangle$ vs temperature $k_{B} T / J$, for $E=0.5 J$. Different system sizes are as in Fig. 1.

$T=T_{c}$. For $L>16, C_{L}$ seems to approach a limit independent of $L$, signaling a breakdown of scaling (in the sense that the corresponding constant $B \approx 0$ ) and hence a finite specific heat $C=\lim _{L \rightarrow \infty} C_{L}$ in the thermodynamic limit, implying that no phase transition takes place. This evidence of the absence of a phase transition is furthermore supported by the cumulant $Q_{L}=\left\langle p_{L}^{2}\right\rangle^{2} /\left\langle p_{L}^{4}\right\rangle$ plotted in Fig. 2, again with $E=0.5 \mathrm{~J}$. For equilibrium Ising models ${ }^{14,15} Q_{c}=0.856$ at the critical point, independent of $L$ for large $L$. Therefore an intersection of different $Q_{L^{-}}$ versus- $T$ curves locates the critical point. Using this equilibrium argument, the missing of the intersection of the different $Q_{L}$ curves is further evidence that the system does not undergo a phase transition. The same trend was observed for larger values of the electric field $E$. Looking at the specific heat $C_{L}$ and cumulant $Q_{L}$, we thus conclude that in the ferromagnetic case the kinetic phase transition reported in the KLS model is destroyed by a simple change in the boundary conditions, which induces a symmetry breaking in the translational invariance of the system.
Antiferromagnetic $(J<0)$ case

For weak $E$, as in equilibrium, the system settles at low temperature into a $(2 \times 2)$ ordering with particles preferentially occupying one of the two sublattices. At high temperature, the system is disordered. However, at sufficiently strong $E\left(\geq E_{c}\right)$, the system becomes disordered at any finite temperature. In the KLS model (i.e., with full PBC imposed), ${ }^{10}$ it has been shown that $E_{c}=2 J$.

Unlike the attractive case, we find that the system with open boundaries still undergoes a kinetically driven phase transition as reported in Ref. 10. Determining the critical temperature as a function of the electric field $E$, we get a phase diagram that, within statistical uncertainty, does not differ from that of the KLS model. It remains to be determined whether the two models have the same critical behavior, such as the values of critical exponents.

\section{CONCLUSION}

We have found that a simple change in the boundary conditions in a driven diffusive system destroys the second-order kinetic phase transition, in the case of attractive interaction. This effect induced by the boundary conditions has no analog in equilibrium systems. In the repulsive case the critical properties are probably unaltered. It would be interesting to investigate how these can be explained in the context of the renormalization-group approach, in terms of the associated fixed-point topology.

\section{ACKNOWLEDGMENTS}

We thank Professor O. G. Mouritsen and Professor R. K. P. Zia for valuable discussions. This work was supported by the Danish Technical Research Council under Grant No. J.nr.16-4296.K. and NSF under Grant No. DMR-8817563.
'S. Katz, J. L. Lebowitz, and H. Spohn, Phys. Rev. B 28, 1655 (1983); J. Stat. Phys. 34, 497 (1984).

${ }^{2}$ H. van Beijeren and L. S. Schulman, Phys. Rev. Lett. 53, 806 (1984).

3J. Marro, J. L. Lebowitz, H. Spohn, and M. H. Kalos, J. Stat. Phys. 38, 725 (1985).

${ }^{4}$ K.-t. Leung and J. L. Cardy, J. Stat. Phys. 44, 567 (1986).

${ }^{5}$ H. K. Janssen and B. Schmittmann, Z. Phys. B 64, 517 (1986).

6 J. L. Valles and J. Marro, J. Stat. Phys. 49, 89 (1987).

${ }^{7}$ M. Q. Zhang, J.-S. Wang, J. L. Lebowitz, and J. L. Valles, J. Stat. Phys. 52, 1461 (1988).

${ }^{8}$ K.-t. Leung, K. K. Mon, J. L. Valles, and R. K. P. Zia, Phys. Rev. Lett. 61, 1744 (1988).

9 J. L. Valles, K.-t. Leung, and R. K. P. Zia, J. Stat. Phys. 56, 43 (1989).

${ }^{10}$ K.-t. Leung, B. Schmittmann, and R. K. P. Zia, Phys. Rev.
Lett. 62, 1772 (1989).

11 Jørgen V. Andersen and Ole G. Mouritsen, Phys. Rev. Lett. 65, 440 (1990); Phys. Scr. T33, 141 (1990); in Phase Transitions in Soft Condensed Matter, edited by T. Riste and D. Sherrington (Plenum, New York, 1989), p. 342; Hans. C. Fogedby and Axel Svane, Phys. Rev. B 42, 1056 (1990).

${ }^{12}$ For some recent works on nonequilibrium systems, see $\mathrm{Y}$. He, C. Jayaprakash, and G. Grinstein, Phys. Rev. A 42, 3348 (1990); H. W. Blöte, J. R. Heringa, A. Hoogland, and R. K. P. Zia, J. Phys. A 23, 3799 (1990); R. Dickman, Phys. Rev. A 41, 2192 (1990); M. Aertsens and J. Nauts (unpublished).

${ }^{13}$ For a review on driven diffusive systems, see B. Schmittmann, Int. J. Mod. Phys. B (to be published).

${ }^{14}$ A. D. Bruce, J. Phys. A 18, L837 (1985).

${ }^{15}$ T. W. Burkhardt and B. Derrida, Phys. Rev. B 32, 7273 (1985). 